\title{
Effects of Bifunctional Chain Extender on the Crystallinity and Thermal Stability of PET
}

\author{
Bo Liu ${ }^{1,2}$, Qianwei $\mathrm{Xu}^{1,2}$ \\ ${ }^{1}$ Key Laboratory of Advanced Civil Engineering Materials of Ministry of Education, Shanghai, China \\ ${ }^{2}$ School of Materials Science and Engineering, Tongji University, Shanghai, China \\ Email: liubo_jay@126.com,xu_qw@mail.tongji.edu.cn
}

Received August 11, 2013; revised September 11, 2013; accepted September 18, 2013

Copyright (C) 2013 Bo Liu, Qianwei Xu. This is an open access article distributed under the Creative Commons Attribution License, which permits unrestricted use, distribution, and reproduction in any medium, provided the original work is properly cited.

\begin{abstract}
Effects of bifunctional chain extender on the crystallinity and the thermal stability of PET were studied in this work. Results of Infrared substractive spectrum indicated that one oxazoline ring of 2,2'-bis(2-oxazoline) (BOZ) reacted with one carboxyl in PET to generate a blocked PET chain with an oxazoline ring, which then reacted with one carboxyl in second PET to form an extended chain. The intrinsic viscosity (IV) of extended PET would increase from 0.61 of initial PET to 0.80 when the addition of $\mathrm{BOZ}$ was $0.52 \mathrm{wt} \%$. Furthermore, when $\mathrm{BOZ}$ was used with hydroxyl-addition-type chain extender 2,2'-(1,4-phenyl)bis(4H-3,1-benzoxazin-4-one) (BNZ) or chain end modifier phthalic anhydride (PA), IV of chain extended PET would be 0.83 and 0.97 respectively. DSC results showed that chain extending would result in a decrease in crystallinity of PET. Comparing with initial PET, the intrinsic viscosity loss of the chain extended products would be smaller after multi-melting processing.
\end{abstract}

Keywords: Poly(Ethylene Terephthalate); Chain Extender; Infrared Substractive Spectrum; Intrinsic Viscosity

\section{Introduction}

Poly (ethylene terephthalate) (PET) is widely used in fibers, bottles and films due to its excellent mechanical strength, chemical and thermal stability [1-3]. A higher molecular weight and lower carboxyl content give PET a better physical and chemical performance, like toughness, hydrolytic and thermal stability $[4,5]$. For each application, therefor, a certain molecular weight is needed. For example, PET for fiber has an $[\eta]$ of 0.65 , for carbonated soft drink bottles, an $[\eta]$ of 0.78 , and for industrial tire cords, an $[\eta]$ higher than $0.85[6]$.

Virgin PET prepared by melt polymerization usually has a low molecular weight because of degradation under molten condition [6]. Besides, recycled PET would undergo a drop in molecular weight or $[\eta]$ caused by thermal and hydrolytic degradation during melting processing. So measures should be taken to obtain higher molecular weight PET. Solid-state polycondensation may overcome this problem. But it is slow and requires special equipment for large-scale operation [6]. A reactive extrusion was commonly practiced, in which compound with di- or multi-functional groups was introduced to react with end groups of PET resulting in chain extend- ing and/or branching [7]. A good chain extender should be compound bifunctional group, easily preparable, thermally stable, nonvolatile in the melt temperature and capable of a fast reaction with polymer chain ends with no by-products [6]. It is desirable for such compound that ring opening or additional reactions could take place between which and PET chain ends, like bis(epoxides) $[8$, 9], bis(cyclic carboxylic anhydride) [10,11], and diisocyanate $[7,12]$.

But These chain extenders introduced undesirable branching or even cross-linking chains [13]. Inata [13-15] claimed that some types of bis cyclic-imino-ethers could be used to prepare linear chain extended PET. Particularly, bis(oxazoline)s could react with PET rapidly, and reduce carboxyl content thus to improve the hydrolytic stability of PET [5].

In the present article, 2,2'-bis(2-oxazoline) (BOZ) was used as the chain extender of PET to increase the intrinsic viscosity and decrease the carboxyl content under melting processing. Infrared substractive spectrum was used to analyze the reaction mechanism. Intrinsic viscosity (IV) and carboxyl content (CV) were measured to evaluate the chain extension effect. $\mathrm{BOZ}$ was combined 
with 2,2'-(1,4-phenyl)bis(4H-3,1-benzoxazin-4-one) (BNZ) and phthalic anhydride(PA) respectively to further increase the IV of PET. The crystallization property was studied by Differential Scanning Calorimetry (DSC). The thermal stability was evaluated under melting processing.

\section{Experimental}

\subsection{Materials}

PET from Shanghai Zili plastic Co., Ltd., with $[\eta]=0.61$, $\mathrm{CV}=21.2 \mathrm{eq} / 106 \mathrm{~g}$. BOZ was prepared in our laboratory, with a purity $>98 \%$. BNZ from Wuhan Jinnuo Chemical Co., Ltd. PA from sinopharm chemical reagent Co., Ltd.

\subsection{Chain Extension Reaction}

Pure PET was vacuum-dried at $130^{\circ} \mathrm{C}$ for $6 \mathrm{~h}$. chain extension reaction was carried out in an internal mixer (Haake90). PET and BOZ were added at the same time. The reaction temperature was set to be $270^{\circ} \mathrm{C}$, and stirring at $50 \mathrm{rpm}$. After $90 \mathrm{~s}$ of melting processing, approximately 3 - $5 \mathrm{~g}$ samples were taken out every once in a while. For each sample, PET-O, PET-1, PET-1.5, PET-2, PET-2.5, PET-3, PET-3.5, PET-4 represent control sample, chain extended samples by 1, 1.5, 2, 2.5, 3, $3.5,4$ times as much BOZ as the theoretical amount respectively.

\subsection{Intrinsic Viscosity Measurement}

Viscosity measurements were conducted at $25.0^{\circ} \mathrm{C} \pm$ $0.1^{\circ} \mathrm{C}$ in an Ubbelohde viscometer on solutions in $60 \% / 40 \% \mathrm{w} / \mathrm{w}$ phenol/tetrachloroethane at a polymer concentration of $5 \mathrm{~g} / \mathrm{L}$.

\subsection{Carboxyl Content Measurement}

The carboxyl content was determined by titrating a standard $\mathrm{KOH} /$ methanol solution into a solution of PET in phenol/tetrachloroethane using Tetrabromophenol Blue as an indicator.

\subsection{FTIR Measurement}

FTIR spectra were performed by using a EQUINOXSS/ HYPER (German) instrument in the range of $500-4000$ $\mathrm{cm}^{-1}$. The samples were dissolved in phenol/tetrachloroethane, washed with ethanol/water to remove the residual chain extenders, the PET particles were powder-pressed with $\mathrm{KBr}$.

\subsection{DSC Measurement}

The calorimetry measurements were performed using a differential scanning calorimeter (DSC-Q100) under nitrogen atmosphere. The samples were heated to $300^{\circ} \mathrm{C}$ rapidly and stayed for $5 \mathrm{~min}$, then cooled to $50^{\circ} \mathrm{C}$ at $10^{\circ} \mathrm{C}$ $/ \mathrm{min}$.

\section{Results and Discussion}

\subsection{Chain Extension Mechanism}

Chain extension reactions lead to a change in the molecular structure of PET, which is not significant respect to the entire molecular chain. Conventional characterization means did not work $[16,17]$. Infrared substractive spectrum was used in this paper and got the desired results shown as Figure 1. $3414 \mathrm{~cm}^{-1}$ arose from -OH stretching vibration, 1699 and $1275 \mathrm{~cm}^{-1}$ due to the vibration of $-\mathrm{COOH}$, the vibration of these two groups showed negative peaks, indicating that the $-\mathrm{COOH}$ concentrations had declined. $1649,1541,1261 \mathrm{~cm}^{-1}$ were the vibration peaks of $\mathrm{O}=\mathrm{CR}-\mathrm{N}-\mathrm{H}$, the peaks were positive, indicating the appearance of amide groups in the chain extension products. 1726,1236 and $1090 \mathrm{~cm}^{-1}$ positive peaks were from $\mathrm{O}=\mathrm{C}-\mathrm{O}-\mathrm{C}$ vibrating, which proved that new ester groups generated during chain extension. It can be inferred that reactions occurred between BOZ and carboxyl groups to form ester and amide groups.<smiles>C1=NCCO1</smiles>

vibration peaks were found at 1649, 1286, 1236, and $1090 \mathrm{~cm}^{-1}$, it indicated the existence of oxazoline rings in the product even after a removal of residual BOZ. From the decrease of carboxyl groups, increase of ester groups, appearance of amide groups, and combined with the increase in intrinsic viscosity, it can be inferred that $\mathrm{BOZ}$ reacted with PET according to Reactions (1) and (2). This result agreed with the reaction model proposed by Inata et al. [13-15].

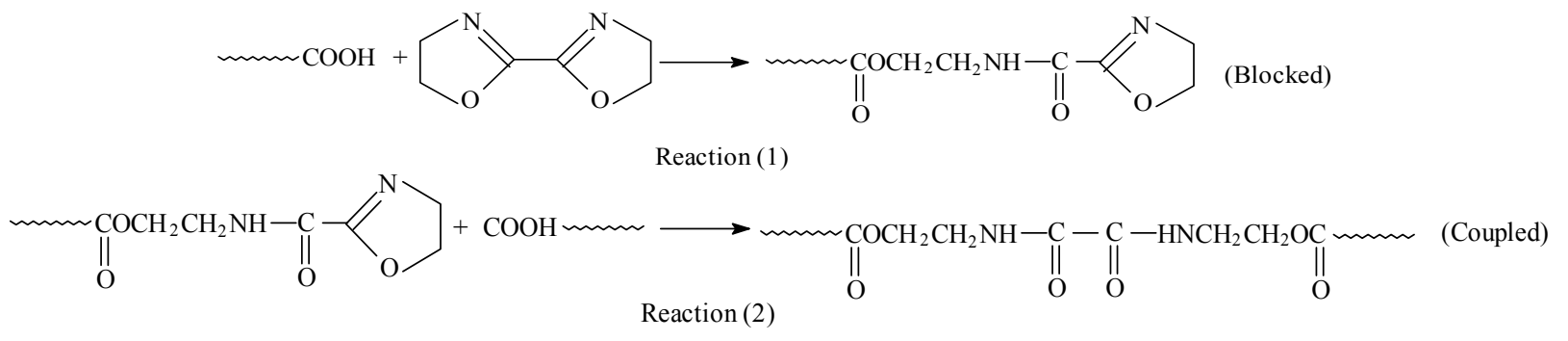




\subsection{The Influence of Added Amount}

Chain extender loss caused by decomposition and volatilization was inevitable, combined with the influence of mixing condition and reaction equilibrium, the actual added amount of chain extender should be larger than the theoretical amount, which was calculated from carboxyl content of pure PET. Table 1 shows the intrinsic viscosity and carboxyl content of each sample processed for $5 \mathrm{~min}$.

As shown in Table 1, IV met a maximum value 0.80 when 3 times as much BOZ as the theoretical amount was used. IV did not always grow with the reduce of carboxyl content, which indicated that not all consumed carboxyl resulted in a chain growth. That is, carboxyl groups did not react with chain extender in a strict molar ratio of $2: 1$, but a portion of $\mathrm{BOZ}$ reacted with only one carboxyl and produced blocked PET. This corresponded with the chain extension mechanism discussed previously.

\subsection{The Influence of Reaction Time}

When the chain extension reaction time is too short, an

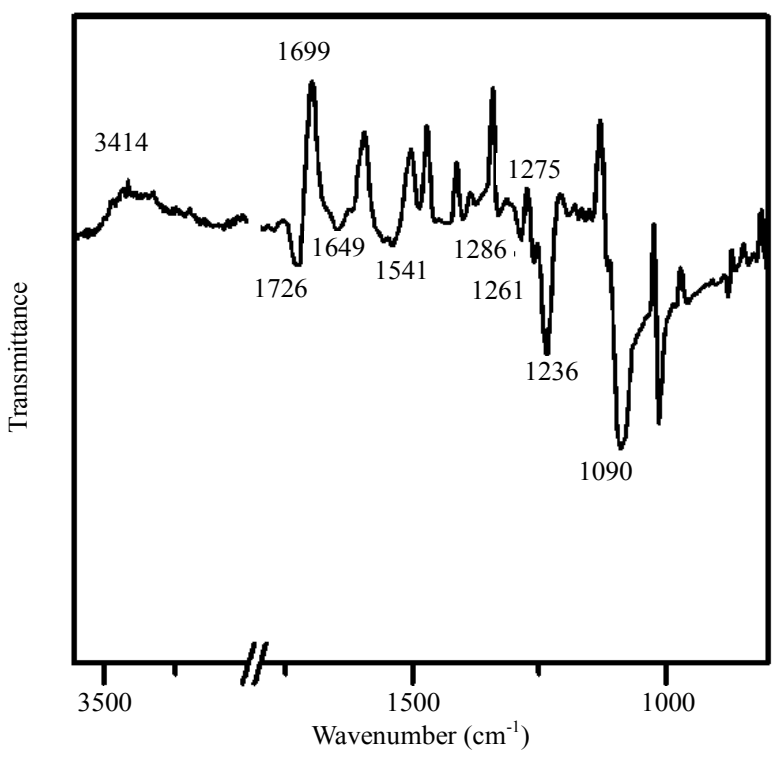

Figure 1. Infrared substractive spectrum of chain extended PET by 0.52 wt \% BOZ and pure PET.

Table 1. IV value and carboxyl content of each sample (5 min processing).

\begin{tabular}{ccc}
\hline Sample & {$[\eta](\mathrm{d} 1 / \mathrm{g})$} & $\mathrm{CV}\left(\mathrm{eq} / 10^{6} \mathrm{~g}\right)$ \\
\hline PET-O & 0.58 & 24.8 \\
PET-1 & 0.67 & 17.5 \\
PET-1.5 & 0.73 & 1.8 \\
PET-2 & 0.76 & 1.5 \\
PET-2.5 & 0.78 & 1.2 \\
PET-3 & 0.80 & 1.0 \\
PET-3.5 & 0.78 & 0.8 \\
PET-4 & 0.77 & 0.8 \\
\hline
\end{tabular}

incomplete reaction would cause a limited increase of molecular weight or IV. If the reaction time is too long, thermal decomposition would be enhanced resulting in an imperfect chain extension result. Figure 2 shows the curves of IV to reaction time. The IV value of each sample came into a balance after 4 - 6 min of chain extension reaction. But there exist some difference according to BOZ added amount. IV values continued to grow within 10 min when BOZ dosage was below 2.5 times as much as the theoretical amount, and the more BOZ was added, the slower its IV value increased. While BOZ added amount exceeded 2.5 times as much as the theoretical amount, IV values showed a trend of decline after the first growth, and the more BOZ was added, the faster it decreased. It was analyzed as follows. The longer time it was processed, the more complete it reacted, then a higher IV value was obtained. Although PET degradation was intensified, the excess $\mathrm{BOZ}$ may react with carboxyl groups newly generated by it, thus eliminated molecular weight loss and gave PET IV value a continuous growth. When BOZ added amount exceeded 2.5 times, Reaction (1) was over promoted and consumed too much carboxyl groups, leading to a cut down of Reaction (2), which was against the molecular weight growth. Then PET IV value began to decrease as thermal degradation was intensified.

Pure PET underwent ongoing degradation in melting state, leading to an increase in carboxyl content as melting time went on (Figure 3). While the carboxyl groups of PET added with BOZ gradually decreased as chain extension reaction proceeded. The more $\mathrm{BOZ}$ was added, the faster carboxyl groups were consumed as Figure $\mathbf{3}$ shows.

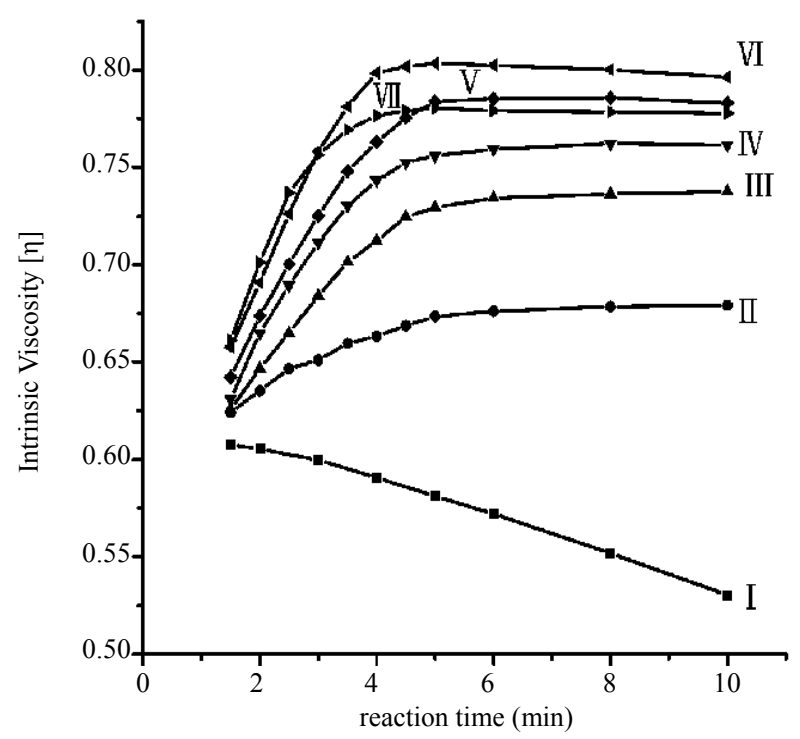

Figure 2. PET IV value varied with reaction time, among which, I-PET-O; II-PET-1; III-PET-1.5; IV-PET-2; V-PET-2.5; VI-PET-3; VII-PET-3.5. 


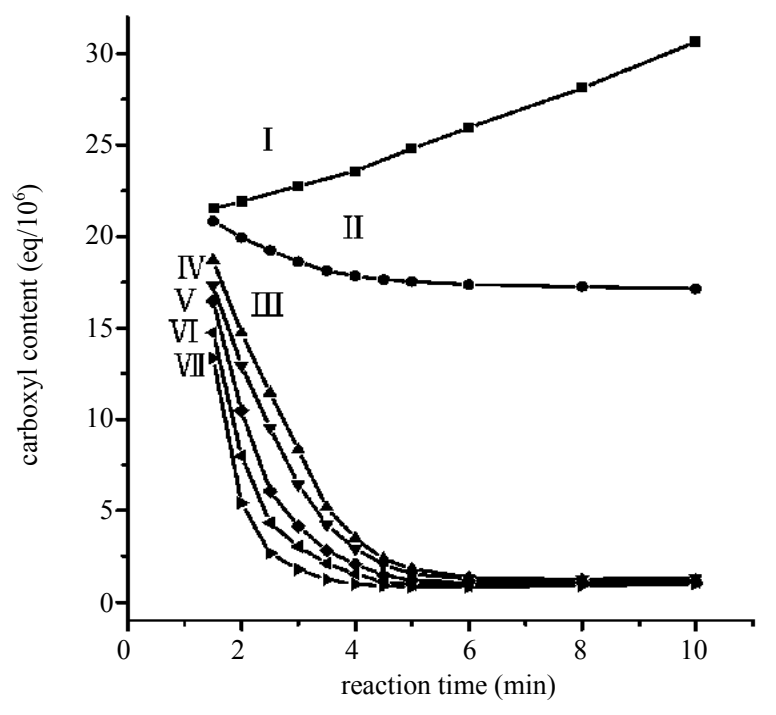

Figure 3. PET carboxyl content varied with reaction time, among which, I-PET-O; II-PET-1; III-PET-1.5; IVPET-2; V-PET-2.5; VI-PET-3; VII-PET-3.5.

\subsection{The Combination of $\mathrm{BOZ}$ and $\mathrm{BNZ}$}

Chain extension effect of BNZ upon PET was valued and shown as Figure 4. When 1.6 wt $\% \mathrm{BNZ}$ was added, $[\eta]$ met a maximum value 0.66 . As previous mentioned, $[\eta]$ met a maximum value 0.80 when 3 times as much $(0.52$ $\mathrm{wt} \%) \mathrm{BOZ}$ as theoretical amount was added. Then 9 samples were prepared accordingly. The IV values of chain extended PET were shown as Table 2. When BOZ and BNZ added amount were $0.52 \mathrm{wt} \%$ and $1.6 \mathrm{wt} \%$ respectively, $[\eta]$ met its maximum value 0.83 , which was higher compared with their singe use. It was confirmed that the combination of carboxyl-addition-type and hydroxyl-addition-type chain extender could further increase the IV of PET.

\subsection{The Combination of $\mathrm{BOZ}$ and $\mathrm{PA}$}

PA can be used to react with PET to generate more carboxyl groups as its high reactivity with hydroxyl group. The reaction formula was shown as follows

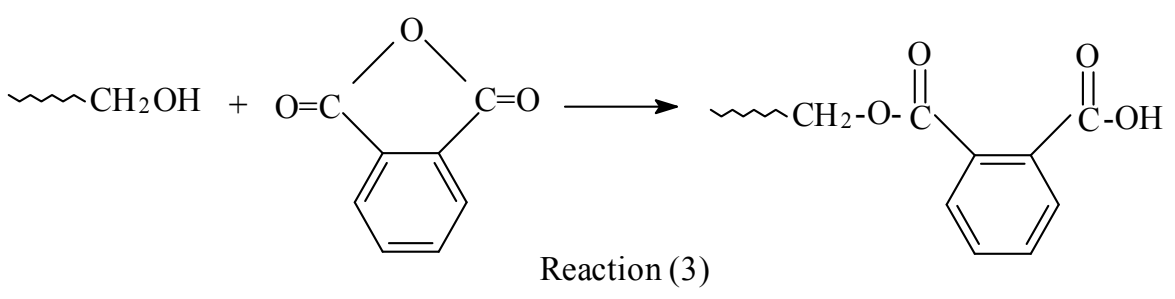

With the increase of carboxyl content, the IV of PET could be increased by BOZ combined with PA.

It can be predicted that the more PA is added, the more carboxyl will be obtained, and the more $\mathrm{BOZ}$ will be needed to obtain a maximum IV value. In order to determine a suitable dosage of PA, various dosage of PA was added into PET, and the carboxyl content was measured as Figure 5 shows.

As shown in Figure 5, PET carboxyl content increased with the increasing dosage of PA, and leveled off when $\mathrm{PA}$ added amount reached $1.1 \mathrm{wt} \%$. Then various dosage of $\mathrm{BOZ}$ was combined with $1.1 \mathrm{wt} \% \mathrm{PA}$ as the chain extender of PET. IV values and carboxyl content were measured as Figure 6 and Figure 7 shows respectively.

As Figure 6 shows, PET reached a maximum IV value 0.80 when $0.52 \%$ wt $\mathrm{BOZ}$ was added in the absence of $\mathrm{PA}$, while the maximum value reached 0.97 when $1.0 \mathrm{wt} \%$ BOZ and $1.1 \mathrm{wt} \%$ PA were added together. The added PA could turn hydroxyl group, which could not react with BOZ, into carboxyl groups, thus to improve the chain extension effect of BOZ and obtain a higher molecular weight chain extended PET. It also showed that under the same BOZ added amount, samples added with PA had higher IV values. As carboxyl content increased, Reactions (1) and (2) were both promoted, leading to a growth in IV. Figure 7 shows PET carboxyl content varied with BOZ added amount. It can be seen that more carboxyl remained under the same BOZ added amount, as the result of increased carboxyl content caused by PA.

\subsection{Crystallization Property}

Table 3 shows the crystallization data of PET-O, PET-1, PET-2, PET-3.5. As shown in Table 3 , the crystallization enthalpy or crystallinity of PET met a decrease after chain extending processing. For PET-O, PET-1 and PET-2, their crystallization enthalpy declined with the increasing of BOZ. This may be resulted by the increase of molecular weight, and several pure PET with different [ๆ] were taken to prove it. As shown in Table 4, the crystallization enthalpy declined with the increase of $[\eta]$, which indicated that the crystallization enthalpy of PET would decline along with the increase of molecular weight.

As shown in Table 1, PET-3.5 had a greater $[\eta]$ value than PET-2, while the crystallization enthalpy of PET-3.5 was higher than that of PET-2. This abnormal phenomenon may caused by the residual chain extender. In order to study the influence of residual BOZ on the crystallization property of chain extended PET, these samples were dissolved, and washed to remove residual BOZ. Their 


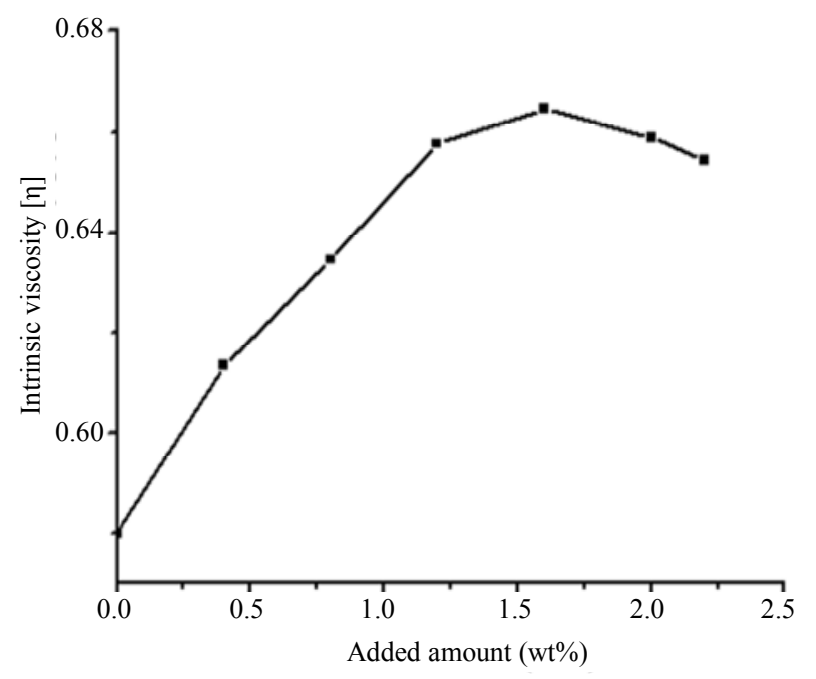

Figure 4. IV of PET versus content of BNZ.

Table 2. PET IV values chain extended by BOZ/BNZ.

\begin{tabular}{llll}
\hline BNZ (wt \%) & 0.43 & 0.52 & 0.61 \\
\hline 1.4 & 0.79 & 0.81 & 0.81 \\
1.6 & 0.81 & 0.83 & 0.81 \\
1.8 & 0.80 & 0.82 & 0.81 \\
\hline
\end{tabular}

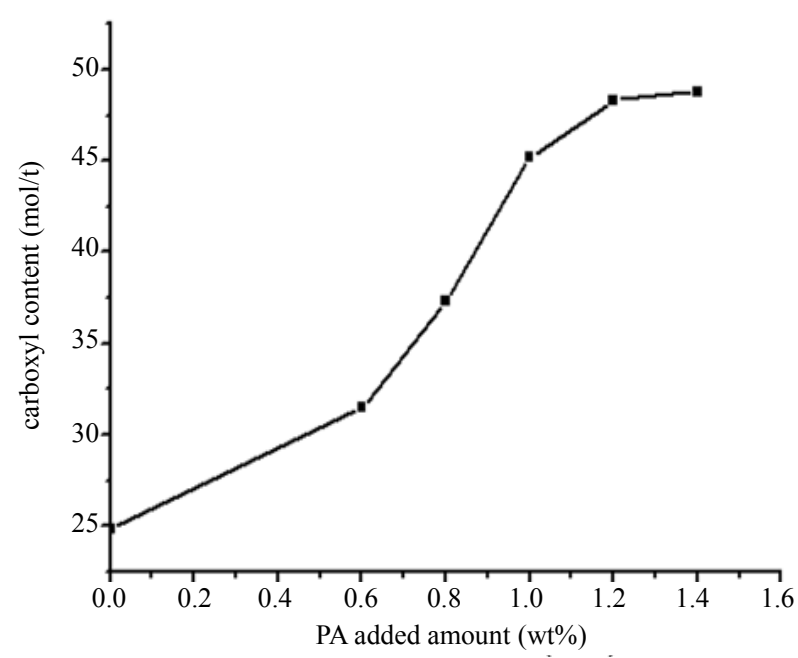

Figure 5. PET carboxyl content varied with PA added amount.

crystallization datum were achieved and shown as Table 3(b). As shown in Table 3, the crystallization enthalpy of PET-1 and PET-2 increased after removal of residual BOZ, while that of PET-3.5 decreased. For chain extended products, there exist un-reacted carboxyl, residual $\mathrm{BOZ}$ and blocked PET, which may react according to the Reactions (1) and (2) under melting process, leading to an increase in molecular weight of PET-1 and PET-2.

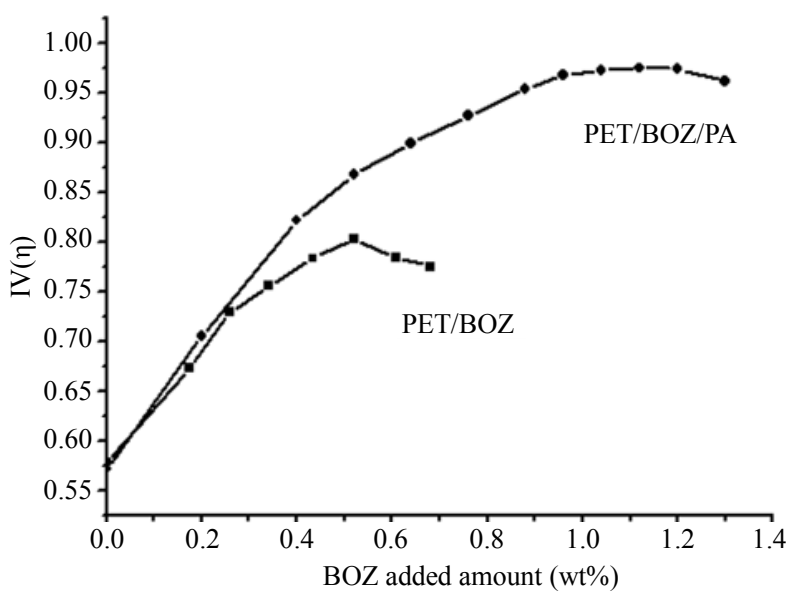

Figure 6. PET IV value varied with $\mathrm{BOZ}$ added amount.

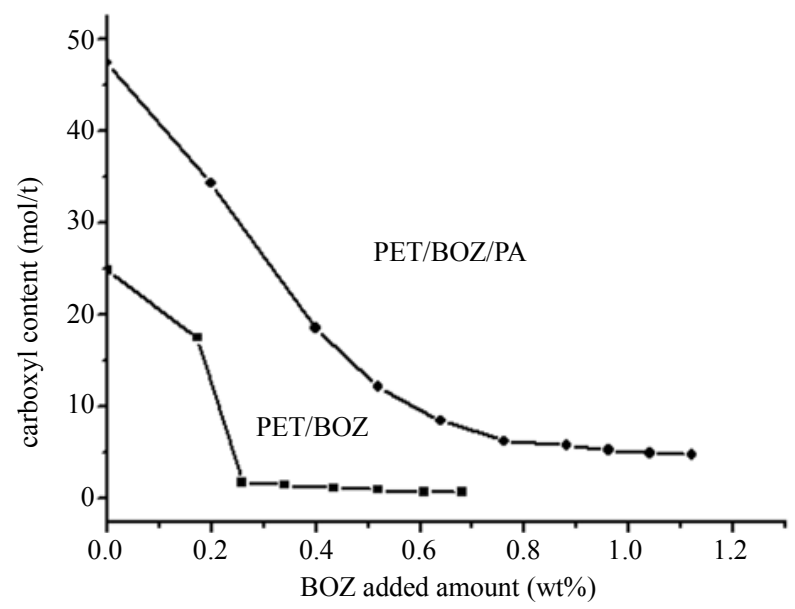

Figure 7. PET carboxyl content varied with BOZ added amount.

Table 3. DSC data of samples before and after removal of residual BOZ (a. unremoved; b. removed).

\begin{tabular}{ccccccc}
\hline Sample & \multicolumn{2}{c}{$\begin{array}{c}\text { Crystallization } \\
\text { enthalpy } / \mathrm{J} / \mathrm{g}\end{array}$} & \multicolumn{2}{c}{ Initial crystallization } & \multicolumn{2}{c}{$\begin{array}{c}\text { Melting } \\
\text { point } /{ }^{\circ} \mathrm{C}\end{array}$} \\
\hline & $\mathrm{a}$ & $\mathrm{b}$ & $\mathrm{a}$ & $\mathrm{b}$ & $\mathrm{a}$ & $\mathrm{b}$ \\
PET-O & 40.22 & 40.26 & 202.62 & 204.01 & 246.84 & 247.46 \\
PET-1 & 38.07 & 38.30 & 199.55 & 203.12 & 245.71 & 246.43 \\
PET-2 & 35.11 & 37.01 & 196.93 & 200.96 & 245.04 & 244.96 \\
PET-3.5 & 37.07 & 35.94 & 203.12 & 203.47 & 247.00 & 245.37 \\
\hline
\end{tabular}

Table 4. Crystallization enthalpy of 4 pure PET varied with [ $\eta$ ].

\begin{tabular}{cc}
\hline$[\eta]$ & Crystallization enthalpy/ J/g \\
\hline 0.53 & 44.03 \\
0.61 & 40.22 \\
0.72 & 37.38 \\
0.81 & 34.66 \\
\hline
\end{tabular}


But for PET-3.5, too much chain extender lead to an excessive consumption of carboxyl groups at Reaction (1), thus to inhibit Reaction (2), making it very small for PET-3.5 molecular weight increase, and the crystallization enthalpy became greater than that of PET-2. While after removing residual BOZ, only Reaction (2) could occur under melting process. For PET-1 and PET-2, Reactions (1) and (2) were both weakened. Then the molecular weight relatively decreased and crystallization enthalpy increased. While for PET-3.5, the removal of residual BOZ avoided carboxyl consumption of Reaction (1) and enhanced Reaction (2), thus to make a relative increase in molecular weight and a decrease in crystallization enthalpy.

\subsection{Thermal Stability}

Melting processing would result in IV loss of PET, accompanied by end groups increase. While DSC results showed that residual chain extender remained reactivity with carboxyl groups, it may help reduce IV loss through reacting with carboxyls newly generated under melting processing, thus to improve thermal stability of chain extended PET. Multi-melting processing was conducted, IV value and carboxyl content were measured. As control, 3 pure PET were chosen and named by R-1, R-2, R-3. Their IV and CV values before and after melting processing were measured and shown in Table 5. The IV losses of chain extended samples were no higher than 0.01, while for pure PET, the IV losses ranged from 0.04 to 0.06 . Then we can come to a conclusion that chain extended samples have better thermal stability.

\section{Conclusions}

1) Infrared substractive spectrum indicated that, after chain extension reaction of PET with BOZ, carboxyl content decreased, ester groups increased and amide groups were generated. One oxazoline ring BOZ reacted

Table 5. IV and CV of samples before and after melting process.

\begin{tabular}{cccccc}
\hline sample & $\begin{array}{c}{[\eta]} \\
\text { (unprocessed) }\end{array}$ & $\begin{array}{c}{[\eta]} \\
\text { (processed ) }\end{array}$ & $\begin{array}{c}\text { IV } \\
\text { loss }\end{array}$ & $\begin{array}{c}\mathrm{CV} /\left(\mathrm{eq} / 10^{6}\right) \\
\text { (unprocessed) }\end{array}$ & $\begin{array}{c}\mathrm{CV} /\left(\mathrm{eq} / 10^{6}\right) \\
\text { (processed })\end{array}$ \\
\hline PET-O & 0.58 & 0.55 & 0.03 & 24.8 & 27.2 \\
PET-1 & 0.67 & 0.69 & -0.02 & 17.5 & 12.8 \\
PET-2 & 0.76 & 0.77 & -0.01 & 1.5 & 1.3 \\
PET-3 & 0.80 & 0.79 & 0.01 & 1.0 & 1.0 \\
PET-4 & 0.77 & 0.76 & 0.01 & 0.8 & 0.8 \\
R-1 & 0.65 & 0.61 & 0.04 & 25.3 & 29.4 \\
R-2 & 0.71 & 0.66 & 0.05 & 50.1 & 57.8 \\
R-3 & 0.78 & 0.72 & 0.06 & 47.3 & 53.1 \\
\hline
\end{tabular}

with one carboxyl in PET to generate a blocked chain with an oxazoline ring, which then reacted with one carboxyl in second PET to form an extended chain.

2) With the addition of $0.52 \mathrm{wt} \% \mathrm{BOZ}$, an improved PET with $[\eta]=0.80$ was obtained, starting from $[\eta]=$ 0.61 . When extra BNZ or PA was added, $[\eta]$ can be increased to 0.83 and 0.97 respectively.

The crystallinity of PET declined after chain extension reaction. Chain extended PET obtained a higher thermal stability over pure samples and met a less IV loss under melting processing.

\section{REFERENCES}

[1] Y. Srithep, A. Javadi, S. Pilla, et al., "Processing and Characterization of Recycled Poly(Ethylene Terephthalate) Blends with Chain Extenders, Thermoplastic Elastomer, and/or Poly(Butylene Adipate-Co-Terephthalate)," Polymer Engineering and Science, Vol. 51, No. 6, 2011, pp. 1023-1032. http://dx.doi.org/10.1002/pen.21916

[2] D. J. Blundell, A. Mahendrasingam and C. Martin, "Orientation Prior to Crystallisation during Drawing of Poly (Ethylene Terephthalate)," Polymer, Vol. 41, No. 21, 2000, pp. 7793-7802.

http://dx.doi.org/10.1016/S0032-3861(00)00128-2

[3] S. S. Pesetskii, B. Jurkowski, O. V. Filimonov, V. N. Koval and V. V. Golubovich, "PET/PC Blends: Effect of Chain Extender and Impact Strength Modifier on Their Structure and Properties," Journal of Applied Polymer Science, Vol. 119, No. 1, 2011, pp. 225-234. http://dx.doi.org/10.1002/app.32532

[4] D. N. Bikiaris and G. P. Karayannidis, "Dynamic Thermomechanical and Tensile Properties of Chain-Extended Poly(Ethylene Terephthalate)," Journal of Applied Polymer Science, Vol. 70, No. 4, 1998, pp. 797-803. http://dx.doi.org/10.1002/(SICI)1097-4628(19981024)70: 4<797::AID-APP20>3.0.CO;2-T

[5] D. N. Bikiaris, G. P. Karayannidis, "Effect of Carboxylic End Groups on Thermooxidative Stability of PET and PBT," Polymer Degradation and Stability, Vol. 63, No. 2, 1999, pp. 213-218. http://dx.doi.org/10.1016/S0141-3910(98)00094-9

[6] G. P. Karayannidis, E. A. Psalida, "Chain Extension of Recycled Poly(Ethylene Terephthalate) with 2,2'-(1,4Phenylene)bis(2-oxazoline)," Journal of Applied Polymer Science, Vol. 77, No. 10, 2000, pp. 2206-2211. http://dx.doi.org/10.1002/1097-4628(20000906)77:10<22 06::AID-APP14>3.0.CO;2-D

[7] Y. Zhang, C. Zhang, H. Li, Z. Du and C. Li, "Chain Extension of Poly(Ethylene Terephthalate) with BisphenolA Dicyanate," Journal of Applied Polymer Science, Vol. 117, No. 4, 2010, pp. 2003-2008. http://dx.doi.org/10.1002/app.32136

[8] D. N. Bikiaris and G. P. Karayannidis, "Calorimetric Study of Diepoxide Chain-Extended Poly(Ethylene Terephthalate)," Journal of Thermal Analysis, Vol. 54, No. 3, 1998, pp. 721-729.

http://dx.doi.org/10.1023/A:1010127500694 
[9] A. A. Haralabakopoulos, D. Tsiourvas and C. M. Paleos, "Chain Extension of Poly(Ethylene Terephthalate) by Reactive Blending Using Diepoxides," Journal of Applied Polymer Science, Vol. 71, No. 13, 1999, pp. 2121-2127. http://dx.doi.org/10.1002/(SICI)1097-4628(19990328)71: $13<2121::$ AID-APP1 $>3.0 . \mathrm{CO} ; 2-\mathrm{Y}$

[10] P. Kiliaris, C. D. Papaspyrides and R. Pfaendner, "Reactive-Extrusion Route for the Closed-Loop Recycling of Poly(Ethylene Terephthalate)," Journal of Applied Polymer Science, Vol. 104, No. 3, 2007, pp. 1671-1678. http://dx.doi.org/10.1002/app.25795

[11] J. S. Forsythe, K. Cheah, D. R. Nisbet, R. K. Gupta, A. Lau, A. R. Donovan, M. S. O'Shea and G. Moad, "Rheological Properties of High Melt Strength Poly(Ethylene Terephthalate) Formed by Reactive Extrusion," Journal of Applied Polymer Science, Vol. 100, No. 5, 2006, pp. 3646-3652. http://dx.doi.org/10.1002/app.23166

[12] N. Torres, J. J. Robin and B. Boutevin, "Chemical Modification of Virgin and Recycled Poly(Ethylene Terephthalate) by Adding of Chain Extenders during Processing," Journal of Applied Polymer Science, Vol. 79, No. 10, 2001, pp. 1816-1824. http://dx.doi.org/10.1002/1097-4628(20010307)79:10<18 16::AID-APP $100>3.0 . \mathrm{CO} ; 2-\mathrm{R}$

[13] H. Inata and S. Matsumura, "Chain Extenders for Polyes- ters. I. Addition-Type Chain Extenders Reactive with Carboxyl End Groups of Polyesters," Journal of Applied Polymer Science, Vol. 30, No. 8, 1985, pp. 3325-3337. http://dx.doi.org/10.1002/app.1985.070300815

[14] H. Inata and S. Matsumura, "Chain Extenders for Polyesters. II. Reactivities of Carboxyl-Addition-Type Chain Extenders; Biscyclic-Imino-Ethers," Journal of Applied Polymer Science, Vol. 32, No. 5, 1986, pp. 5193-5202. http://dx.doi.org/10.1002/app.1986.070320534

[15] H. Inata and S. Matsumura, "Chain Extenders for Polyesters III, Addition-Type Nitrogen-Containing Chain Extenders Reactive with Hydroxyl End Groups of Polyesters," Journal of Applied Polymer Science, Vol. 32, No. 4, 1986, pp. 4581-4594. http://dx.doi.org/10.1002/app.1986.070320423

[16] C. R. Nascimento, C. Azuma, R. Bretas, M. Farah and M. L. Dias, "Chain Extension Reaction in Solid-State Polymerization of Recycled PET: The Influence of 2,20-Bis2-oxazoline and Pyromellitic Anhydride," Journal of Applied Polymer Science, Vol. 115, No. 6, 2010, pp. 31773188. http://dx.doi.org/10.1002/app.31400

[17] R. Dhavalikar and M. Xanthos, "Monitoring the Evolution of PET Branching through Chemorheology," Polymer Engineering and Science, Vol. 44, No. 3, 2004, pp. 474-486. 